\title{
Microstructural changes of the mucous membrane of the fallopian tube during prolonged opioid exposure
}

\author{
M. V. Podolyuk, Ya. T. Ivankiv, L. R. Mateshuk-Vatseba \\ Danylo Halytsky Lviv National Medical University \\ Corresponding author. E-mail: mariapodolyk1979@gmail.com
}

Paper received 28.12.19; Accepted for publication 16.02.20.

\section{https://doi.org/10.31174/SEND-NT2020-224VIII27-10}

\begin{abstract}
The article presents data on structural and functional changes of the mucous membrane of the uterine tube in the dynamics of two-, four- and six-week exposure to nalbuphine. The material of the study were sexually mature rats-females of the line "Wistar" in the amount of 26 animals weighing 180-220 g, aged 3.5-4 months. Animals were injected with nalbuphine intramuscularly, daily, once a day, at the one-time interval. Histological preparations were prepared according to the conventional method. The animals were kept in a vivarium, the work was carried out following the "Rules of work using experimental animals". Before sampling, the animals were primed with an intraperitoneal injection of thiopental (at a rate of $25 \mathrm{mg} / \mathrm{kg}$ ), then an anterior abdominal wall was opened, followed by removal of the fallopian tube. During the experimental research, changes in the structure of the mucous membrane of the fallopian tube were established. The first signs were found within 2 weeks of the experimental effect of nalbuphine: swelling of the mucous membrane of the fallopian tube, slight smoothing of the folds and local desquamation of the epithelium, moderate infiltration of lymphocytes of the mucous membrane. Vascular microcirculatory vessels of the mucous membrane are slightly expanded. After 4 weeks of the experiment, degenerative and inflammatory changes in the uterine tube wall and manifested by swelling and polymorphocellular infiltration of the membranes of the wall of the test organ wall, an increase in the number of altered epitheliocytes, vessels are hyperemic, overflowing with aggregated erythrocytes, sometimes containing neutrophilic granulocytes and lymphocytes. After 6 weeks of administration of nalbuphine, destructive changes in the cells of the mucous membrane of the fallopian tube and the links of its hemomicrocirculatory bed accrue, manifested by desquamation of the epithelium of the mucous membrane, infiltration of the mucous membrane by lymphocytes, there is an expansion of the lumen of the vessels of the microcirculatory bed and filling the lumen of the blood plasma, there are lashes of different heights, the characteristic grouping of them into conglomerates.
\end{abstract}

Keywords: microstructure, fallopian tube, opioid, experiment.

Introduction. In modern medicine, opioid analgesics have long been used to relieve acute pain. Research on structural changes in various organs and tissues with short-term and long-term effects on the body of opioid analgesics is relevant $[1,2,3$,$] . Nalbufin (nubain) is a narcotic analgesic, a$ semi-synthetic opioid, a derivative of phenanthrene. It is similar in structure to morphine and naloxone, belongs to opioids of mixed (agonist-antagonistic) action. The problem of opioid addiction has become a widespread epidemic process. In this regard, an anti-narcotic strategy for overcoming the problem of polyetiological pathology has been developed in European countries. Predominantly, studies related to social, legal, and psychological aspects, not paying attention to the fact that significant metabolic and structural changes, early disability and mortality of patients with opioid dependence require the study of pathogenetic mechanisms of development and progression of multiple organ comorbid conditions [4]. The development of pharmacotherapy for narcotic substances, among which the opioid such as nalbuphine is increasingly used, requires the development of measures for the prevention and correction of side effects and complications [5]. One of the important directions in modern obstetric anesthesiology is the choice of medicines that are closest to their own pain (endorphin) system [6]. These drugs include opioids. In the professional literature there are data on the effect of opioids on the vascular membrane of the eyeball, microstructural changes in the tongue, thymus, spleen, pancreas, myocardium, nervous system, retina have been investigated $[4,7,8,9,15]$. However, almost no information about the mechanisms of action of opiates and opioids on the structure and angioarchitectonics of the female reproductive system, including the mucous membrane of the fallopian tube.

The aim of the study. To establish peculiarities of microstructure of the uterine mucosa of the white rat in the normal state and changes in the structural organization of the mucous membrane of the uterine tube due to the introduction of opioids in the experiment.

Materials and methods of research. The study was performed on 26 sexually mature white female rats aged $3.0-4.5$ months and body weight $180-220 \mathrm{~g}$. The microstructural organization of the uterine mucosa in the normal state was studied in 5 animals. The experimental animals were divided into 3 groups. The first group of test animals (5 rats) were intramuscularly injected with nalbuphine once a day, daily for 2 weeks (once a week $-8 \mathrm{mg} / \mathrm{kg}$, II $15 \mathrm{mg} / \mathrm{kg}$ ), the second group (5 rats) was administered intramuscularly for 4 weeks (I week - $8 \mathrm{mg} / \mathrm{kg}$, II - $15 \mathrm{mg} / \mathrm{kg}$, III - $20 \mathrm{mg} / \mathrm{kg}$, IV - $25 \mathrm{mg} / \mathrm{kg}$ ); the third group (5 rats) intramuscularly injected nalbuphine for 6 weeks (I week - 8 $\mathrm{mg} / \mathrm{kg}$, II - $15 \mathrm{mg} / \mathrm{kg}$, III - $20 \mathrm{mg} / \mathrm{kg}, \mathrm{IV}-25 \mathrm{mg} / \mathrm{kg}$, $\mathrm{V}-30 \mathrm{mg} / \mathrm{kg}$, VI - $35 \mathrm{mg} / \mathrm{kg}$ ) [8]. Control - 6 white female rats, which were administered the physiological solution. Material for examination is presented by histological preparations of the mucous membrane of the fallopian tube. For histological examination, sections of the uterine mucosa were stained with hematoxylin and eosin. The preparations were examined using a Leica DM $2500 \mathrm{mi}-$ croscope and photographed with a Leica DFC $450 \mathrm{C}$ camera at microscope magnifications: x200, x400; x1000. All animals were kept in a vivarium in the Danylo Halytsky Lviv National Medical University. The experiments were carried out in accordance with the conditions of the European Convention for the Protection of Vertebrate Animals, which are used for experimental and other scientific purposes (Strasbourg, 1986), Council of Europe Directive 86/609 / EEC (1986), Law of Ukraine No. 3447-IV “On the Protection of Animals of the Common Principles of Animal Experimentation, approved by the First National Congress of Ukraine on Bioethics (2001). 
Results of the research. Normally, the uterine tube of rats-females has a typical structure: it consists of three parts that are localized outside the uterus and one part, known as an intrauterine, located in the uterine wall. All parts have different diameters; the funnel is the widest part and the intrauterine part is the narrowest. (Fig. 1A).

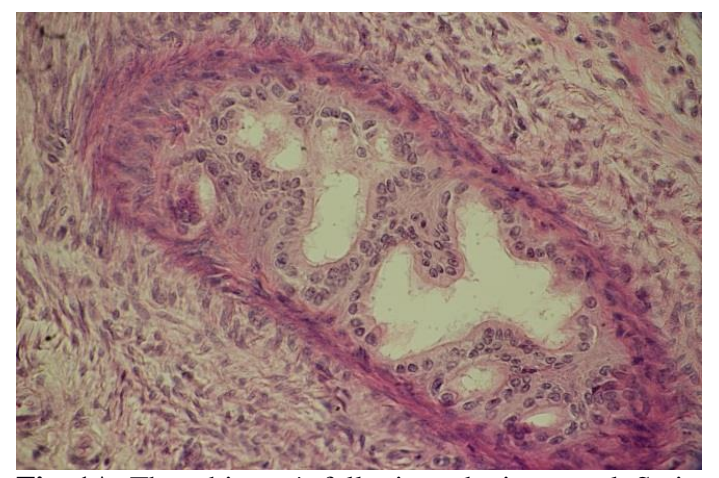

Fig. 1A. The white rat's fallopian tube is normal. Stained with hematoxylin and eosin. Microphoto. Coll. : x 400.

The longest fimbria of the funnel of the uterine tube of the female rat is contacted with the ovary. The uterine wall is formed by three membranes (mucous, muscular and serous). The mucous membrane is represented by the epithelium and its own lamella. The fallopian tube epithelium is a pseudo-multilayer (ciliated simple columnar epithelium) located on the basement membrane. Among the epitheliocytes distinguish high prismatic cells, on the apical surface of which the cilia are identified, there are secretory cells (tubular exocrinocytes) between the ciliary cells, intermediate cells, and cells that do not reach the lumen of the fallopian tube - basal cells. The lamina propria of the mucous membrane is formed by a loose connective tissue, which contains the fibrous structures and cellular elements inherent in the connective tissue, as well as the vessels of the microcirculatory bed, nerve fibers. The mucous membrane of the fallopian tube forms longitudinal folds, which are the most branched and highest in the funnel portion and the least branched and lowest in the intrauterine part. The muscular sheath is formed by two layers of smooth myocytes, between which are layers of connective tissue. The outer lining of the fallopian tube is serous, covered with a singlelayer squamous epithelium - mesothelium. All layers of the fallopian tube are well vascularized. The hemomicrocirculatory bed of a female white rat contains arterioles, precapillary arterioles, capillaries, postcapillary venules, venules. The first morphological changes in the structure and angioarchitectonics of the uterine mucosa were detected after 2 weeks of administration of the drug Nalbufin to rats (Fig. 2A, Fig. 2B). In the internal uterine part of the fallopian tube there is edema of the mucous membrane, smoothing of the folds and local desquamation of the epithelium. The vessels of the microcirculatory bed of the mucous and serous membranes are filled with erythrocyte-leukocyte aggregates adjacent to the wall of the endothelium. In the isthmus of the fallopian tube (Fig. 2B), there is also swelling of the mucous membrane, desquamation of the epithelium, infiltration by lymphocytes of the own lining of the mucous membrane. Perivascular edema was noted in some places.

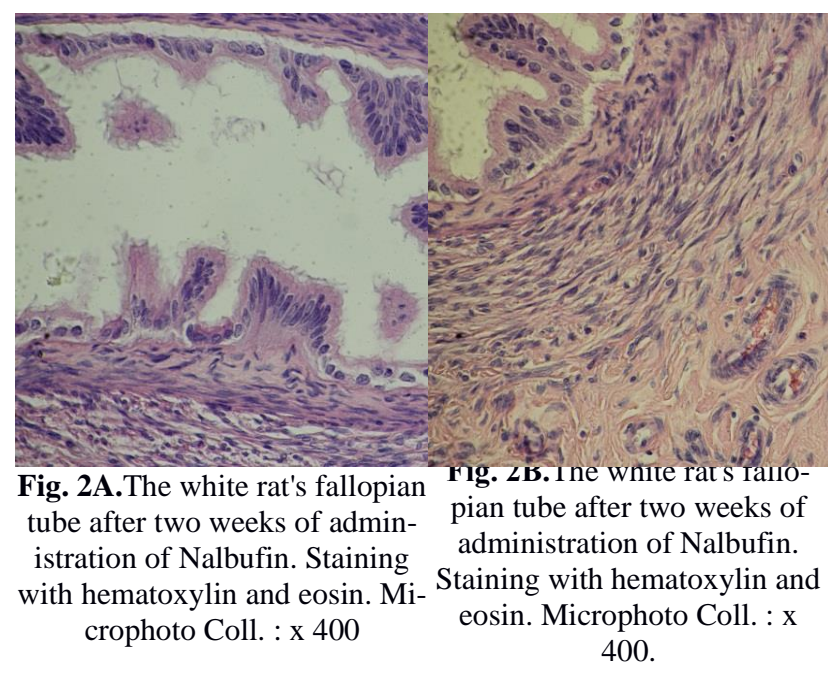

The cytoplasm of some ciliary cells is enlightened. The expressed changes are observed in vessels of a microcirculatory bed, in particular vessels of lamina propria of a mucous membrane are expanded, overflowed with erythrocytes, in some places contain neutrophilic granulocytes and lymphocytes. Erythrocyte aggregation is observed in some capillaries. The main substance of connective tissue around the hyperemic vessels is somewhat swollen, infiltrated by lymphocytes. Four weeks after the administration of nalbuphine, we documented increasing changes in the destructive nature of the mucosa of the uterine tube in all its parts. In the internal uterine part, as in the previous term, the desquamation of the epithelium, local smoothing of the folds expanding the lumen of the tube in this segment, moderate infiltration by lymphocytes of the own lamina (Fig. 3A) is observed. In the ampoule part, there is noticeable swelling of the mucous membrane, detachment of the epithelium from its own lamella, the remains of cellular detritus in the lumen of the tube (Fig. 3B).

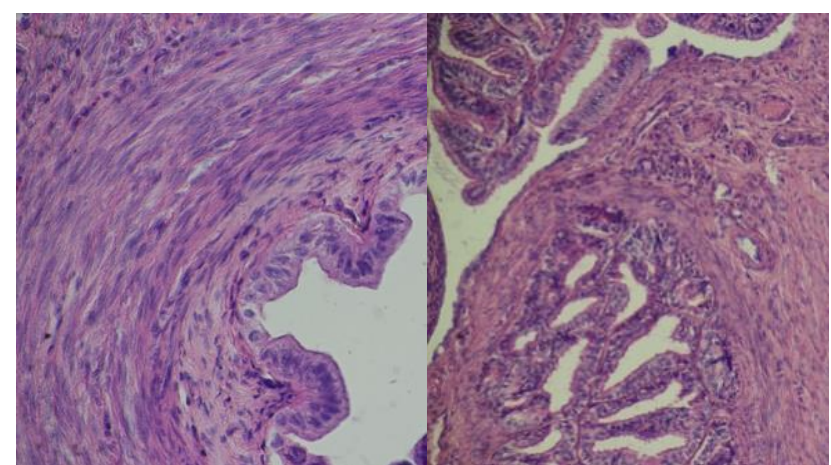

Fig. 3A. The white rat's fallo- Fig. 3B. The white rat's fallopian tube after four weeks of ad- pian tube after four weeks of ministration of Nalbufin. Stain- administration of Nalbufin. ing with hematoxylin and eosin. Staining with hematoxylin and Microphoto Coll. : x $400 . \quad$ eosin. Microphoto Coll. : x 200.

The expressed changes are registered also in vessels of a microcirculatory bed. The vessels of the lamina propria of the mucous membrane of the fallopian tube are dilated, overflowing with erythrocytes, sometimes containing neutrophilic granulocytes and lymphocytes. After six weeks of the administration of nalbuphine, deep changes of destructive nature was observed in the uterine tube, namely, the absence of folds in certain areas, desquamation of the epithelium, and thickening of the own lamina (Fig. 4A, Fig. 4B). Similar changes are present in the isthmus part, also, 
there is an expansion of the lumen of the vessels of the microcirculatory bed and filling their lumen with blood plasma. In the ampoule part, there are lashes of different heights, characterized by their grouping into conglomerates (Fig.4B). Areas with no basal cells are observed. Cilia cells and secretory cells have a modified shape. The cytoplasm of cells is narrow, unevenly colored. The secretory cells are located far from each other. The mucous membrane of the fallopian tube is of unequal thickness, indicating focal necrotic changes in its cells. In the vessels of the lamina propria of the mucous membrane, small veins, and venules, and to a lesser extent, the arteries and arterioles are hyperemic, overflowing with erythrocytes. In some capillaries, there is an aggregation of red blood cells, indicating the development of stasis.

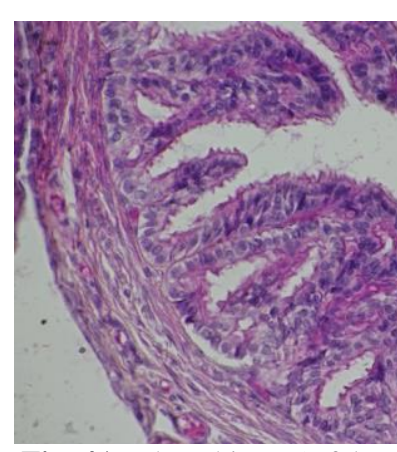

Fig. 4A. The white rat's fallopian tube after six weeks of administration of Nalbufin. Staining with hematoxylin and eosin. Micro photo Coll. : x 400

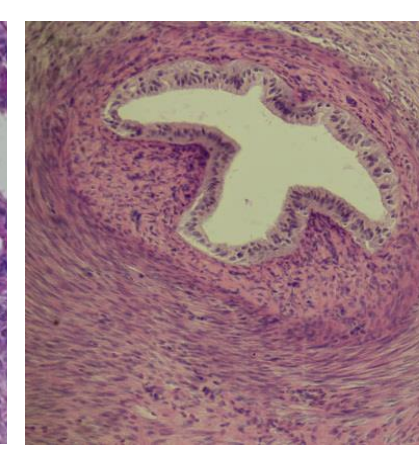

Fig. 4B. The white rat's fallopian tube after six weeks of administration of Nalbufin. Staining with hematoxylin and eosin. Micro photo Coll. : x 1000.
Discussion of the results. The vast majority of authors note the development of profound changes in angioarchitectonics of the organ with prolonged exposure to nalbuphine $[12,13,14]$. Thus, the destructive restructuring of the links of the microcirculatory bed of the eyeball, pancreas, colon wall, cerebellar cortex has been described under conditions of 6 -week administration of nalbuphine $[15,16,17$, 18]. This is confirmed by our observations: after 6 weeks of the experiment, there are changes in the vessels of the microcirculatory bed: signs of stagnation, plethora, arterioles wall are unevenly thickened, twisted, with phenomena of hyalinosis, venules are sharply expanded. Most diseases of the fallopian tubes arise as a result of inflammatory processes that lead to complete or partial obstruction of the fallopian tubes due to infiltration, obliteration of their walls. In the professional literature it is stated that the most common causes of anatomical disorders may be: post-operative surgery on internal genital organs, postpartum complications, endometriosis, etc. Among the etiological factors of inflammatory processes in the pelvic organs, a special role belongs to $\mathrm{S}$. trachomatis, which causes the inflammatory process in the fallopian tubes, which leads to their occlusion due to the destruction of the fibrils and the formation of the hydrosalpinx, and promotes the development of inflammatory reaction around the tubes accompanied by mobility. , normal capture and promotion of the ovum. The causative agent of gonorrhea - Neisseria gonorrhoeae causes the development of the inflammatory process and promotes the formation of adhesions in the pelvis. Attached to the cells of the epithelium of the fallopian tubes, these microorganisms have a toxic effect and cause narrowing or complete obliteration of the tubes, which significantly impairs the promotion of the egg cell into the uterine cavity and there are prerequisites for the development of ectopic pregnancy [10]. The results of our study showed that with prolonged exposure to opioids, pathological processes develop in the fallopian tube, its mucous membrane suffers changes. Six-week administration of nalbuphine causes the restructuring of the uterine tube structural organization, such as in long-term inflammatory processes. In particular, the lumen of the fallopian tubes increases, the ciliated cells are desquamated and completely replaced by the secretory ones, the adenomatous proliferation of the tubular epithelium occurs. The stroma of the mucous membrane is impregnated with inflammatory infiltrate. The lamina propria of the mucous membrane is made of fibrous tissue. The folds of the mucous membrane of the uterine tube are thickened, the luminescence of the vessels of the microcirculatory bed is full-blooded. In venules and capillaries, the marginal standing of polymorphonuclear leukocytes is noted. The endothelial plate in most of the capillaries is destroyed and incomplete. Around the vessels, there is interstitial edema [11].

Conclusions. 1 . The first signs of infraction of the structure of the mucous membrane of the uterine tube of the white female rat were detected after 2 weeks of experimental effects of nalbuphine: swelling of the mucous membrane of the fallopian tube, slight smoothing of the folds and local desquamation of the epithelium, moderate infiltration of the lymphocytes of the mucous membrane. The vessels of the microcirculatory bed of the mucous membrane are slightly expanded. 2. After 4 weeks of the experiment, degenerative-inflammatory changes in the wall of uterine tube accrue and manifest themselves with swelling and polymorphocellular infiltration of the membranes of the organ wall, an increase in the number of altered epitheliocytes, vessels hyperemic, overflowing with aggregated erythrocytes, sometimes containing neutrocytes. 3. After 6 weeks of administration of nalbuphine, there increase destructive changes in the cells of the mucous membrane of the fallopian tube and the links of its microcirculatory bed, manifested by desquamation of the epithelium of the mucous membrane, infiltration of the mucous membrane of the lymphocyte, filling their lumen with blood plasma, there are lashes of different heights, characteristic of grouping them into conglomerates.

Prospects for further research are to find the most effective methods for correlating structural changes in the organs of the female reproductive system caused by the use of drugs. 


\section{REFERENCES}

1. Holovats'kyy A.S., T.V. Harapko T.V. "Structural changes in organs and tissues during action on the body of opioid analgesics". Uzhgorod University Scientific Bulletin, series «Medicine», issue 2 (52), 2015 p.130-134.

2. Klimenko N. A. Sorokina I. V., Savenko I. A. "Morphofunctional state of the thymus and spleen during inflammation against the background of the action of the non-selective blocker of opioid peptides naloxone. Experimental and clinical medicine". №. 1, 2010 pp. 10-15.

3. Mateshchuk-Vatseba L. R. Diskovs'kyy I. S. "Ultrastructural changes in the skin of rats with long-term opioid exposure Bulletin of the Ukrainian Medical Dental Academy "Actual problems of modern medicine". Vol.14, №. 4 (48). 2014, pp. 205 208.

4. Pidval'na U.YE. Structural features of the eyeball under conditions of long-term opioid exposure in the experiment. Bulletin of the Ukrainian Medical Dental Academy "Topical problems of modern medicine". V.14. № 4 (48). 2014 S. 209-212.

5. Buhayevs'kyy K. A. "Practical use of a complex of methods of non-drug recovery after surgical treatment of impaired ectopic pregnancy". South Ukrainian medical scientific journal. № 20. 2018, Art.20-22

6. Kiselev A.G., Zaitsev A.A. The mechanisms of the influence of opioid analgesics on the reaction of the endogenous opiate system of the body and the regulation of contractile activity of the uterus with a nociceptive response in an experiment in rats in a model of induced labor pain. Journal of Obstetrics and Women's Diseases. 2015; 64 (3). S.11-17.

7. Zin'ko A.V. "The circulatory bed of the rat radiant crown is normal and subject to long-term opioid exposure". Zaporizhzhia medical journal. No. 3 (90). 2015 Pp. 78-81.

8. Holovats'kyy A.S., Val'ko O.O., Volkov K.S., Kramar S.B. "Submicroscopic changes of lymph nodes of iliac lymph nodes in the dynamics of chronic opioid exposure". Clinical anatomy and surgical surgery. T.17. \# 1, 2018 Pp. 35-40.
9. Zhylka N. YA., Myronyuk I. S., Slabkyy H. O. "Characteristics of some indicators of reproductive health of the female population of Ukraine". Wiad Lek, 71, 9, 1803-1808. 2018 Art. 1803-1808

10. Fylypyuk D. O. "Chronic viral diseases as a cause of inflammation of female genital organs and infertility". ISSN $2411 \square 4944$. Topical issues of pediatrics, obstetrics, and gynecology. №2. 2016

11. Holubovs'kyy I. A. "Dynamics of morphological changes in the tissues of the fallopian tubes in acute and chronic inflammation in women of reproductive age." BIOMEDICAL AND BIOSOCIAL ANTHROPOLOGY 2013, No21).

12. Radchenko T.M. "Gender features of prevalence and clinical and psychopathological manifestations of opioid dependence in women. Ukrainian Bulletin of Psychoneurology." T. 24, no. 2. 2016 Pp. 78-81.

13. Pokotylo V. YU., Lohash M. V. "Morphometric analysis of changes in the mitochondrial apparatus of the white rat heart myocardium under experimental conditions. Bulletin of problems in biology and medicine", 2018 - cyberleninka.ru

14. Pat. No. 76564 U Ukraine, IPC A 61 K $31 / 00$ Method for modeling physical opioid dependence in rats. Applicants: Onys'ko R.M., Pal'tov YE.V., Fik V.B., Vil'khova I.V., Kryvko YU.YA., Yakymiv N.YA., Fit'kalo O.S; patentee: Danylo Halytskyi Lviv National Medical University. - №u201207124; claimed 12.06.2012; publ. 01/10/2013 Bul. №1.

15. Paltov Y., Kryvko Y., Fik V. et al. Dynamics of the onset of pathological changes in the retinal layers at the end of the first week of opioid exposure. Deutscher Wissenschaftsherold. German Science Herald. №2. - P. 2016p. C.30-33

16. Kryvko YU.YA., Khres'ko N.I. "Ultrastructural reconstruction of the colon wall with chronic opioid (nalbuphine) exposure in an experiment." Uzhgorod University Scientific Bulletin, series «Medicine», issue 2 (56), 2017.

17. Bekesevych A.M. "Morphological features of the cerebral cortex structure in normal and long-term opioid exposure." Zaporizhzhia medical journal. 2015. No. 3 (90). P. 82 